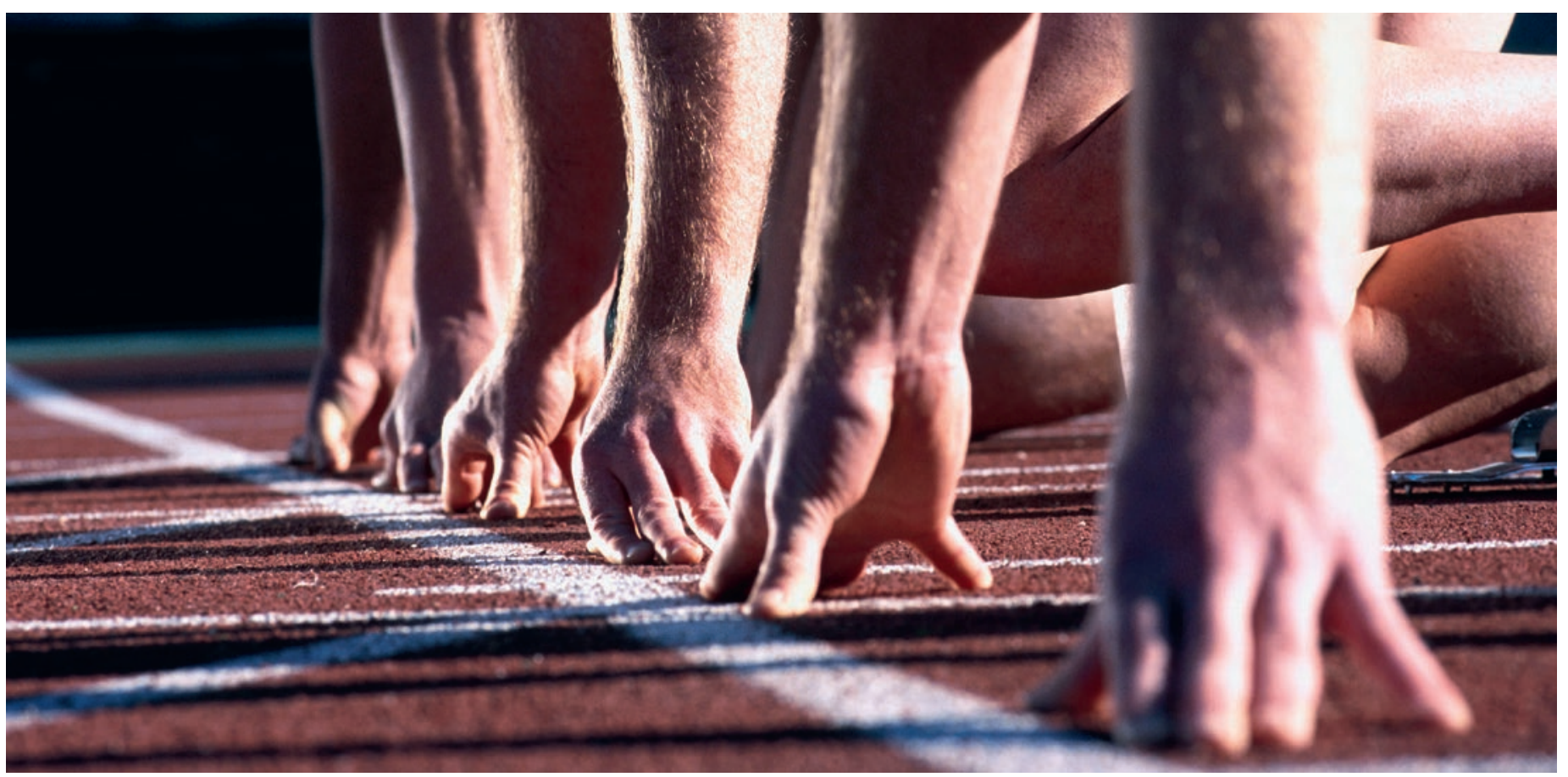

\title{
Antidoping Schweiz wird Swiss Sport Integrity
}

\section{Carina Brunner}

Eidg. dipl. Apothekerin, Stiftung Swiss Sport Integrity, Bern

Die nationale Antidoping-Organisation heisst nun Swiss Sport Integrity. Zum Jahreswechsel wurden Aufgabengebiet und Stiftungszweck von Antidoping Schweiz erweitert und der Namenswechsel beschlossen. Zudem gelten seit dem 1. Januar 2022 strengere Antidoping-Regeln für Glukokortikoide.

Höchstleistung, Freundschaft und Respekt: Die olympischen Werte prägen weltweit den Sport und spielen auch im Alltag eine wichtige Rolle. Jedoch wurden seit mehreren Jahren immer wieder Vorwürfe zu unethischen Trainingsmethoden im Schweizer Sport laut. Die Verantwortung lag bisher bei den Sportverbänden selbst, Fehlverhalten und Missstände in der eigenen Organisation zu identifizieren und gegebenenfalls zu sanktionieren.

Diese Verantwortung wurde nun übertragen, und mit Swiss Sport Integrity hat der Schweizer Sport eine unabhängige und sichere Anlaufstelle erhalten, damit Ethikverstösse und Missstände schnellstmöglich aufgedeckt und beseitigt werden. Ausserdem führt Swiss Sport Integrity die Aufgaben und Tätigkeiten von Anti- doping Schweiz weiter und fungiert weiterhin als nationale Antidoping-Organisation.

\section{Meldeportal eingerichtet}

Das Meldeportal von Swiss Sport Integrity unter www. sportintegrity.ch steht allen Personen offen, die eine Meldung über mögliche Verstösse oder Missstände machen wollen. Bei einer Erstberatung wird über die Vorgehensmöglichkeiten informiert und gegebenenfalls für eine vertiefte Beratung an eine geeignete Beratungsstelle vermittelt.

Die eingegangene Meldung wird geprüft und wenn angebracht an die Strafverfolgungsbehörden weitergeleitet. Bei Zuständigkeit von Swiss Sport Integrity wird 
eine Untersuchung durchgeführt, und ein Schlussbericht zuhanden der Disziplinarkammer des Schweizer Sports (DK) verfasst, welche über mögliche Sanktionen befindet.

\section{Strengere Regeln für Glukokortikoide}

Für die Dopingliste 2022 wurden von der Welt-AntiDoping-Agentur (WADA) Anpassungen mit Auswirkungen auf den Dopingstatus von Arzneimitteln vorgenommen. Diese betreffen insbesondere Glukokortikoide. Zusätzlich zu deren oraler, intramuskulärer, intravenöser und rektaler Anwendung sind neu jegliche Injektionen im Wettkampf verboten. Ist eine Substanz «im Wettkampf» verboten, gilt u.a. deren Anwendung in dieser Zeitperiode oder deren Nachweis bei einer Dopingkontrolle, die «im Wettkampf» stattfindet, als Verstoss gegen die Antidoping-Bestimmungen. Ausgenommen davon sind Fälle, in denen eine Ausnahmebewilligung zu therapeutischen Zwecken (ATZ) vorliegt oder nachträglich erteilt werden kann.

Das Verbot wurde erweitert, da lokale Injektionen von Glukokortikoiden zu vergleichbaren Plasma- und Urinkonzentrationen wie eine systemische Verabreichung

Für die Dopingliste 2022 wurden Anpassungen mit Auswirkungen auf den Dopingstatus von Arzneimitteln vorgenommen.

von Glukokortikoiden führen können. Entsprechend kann für die lokalen Injektionen von einer vergleichbaren potenziellen Leistungssteigerung und von vergleichbaren gesundheitlichen Risiken ausgegangen werden [1]. Andere Verabreichungswege (z.B. inhalativ, topisch) von Glukokortikoiden bleiben im und ausserhalb des Wettkampfes erlaubt.

Mit Hilfe der Medikamentenabfrage Global DRO (via www.sportintegrity.ch/medikamente oder Medi-Check App) kann der Dopingstatus von Arzneimitteln unter Berücksichtigung des Verabreichungswegs überprüft werden.

\section{Auswaschphasen beachten}

Die Zeitdauer von der Anwendung eines Glukokortikoides bis zu dessen Ausscheidung aus dem Körper ist abhängig von der Substanz, dem Verabreichungsweg und der Dosierung. Dies bedeutet, dass nach Anwendung eines Glukokortikoides während einer unterschiedlich langen Zeitspanne eine positive Dopingprobe bei einer Dopingkontrolle im Wettkampf möglich ist. Die WADA tenen Glukokortikoide definiert [2]. Die jeweiligen pro Substanz und Verabreichungsweg geltenden Auswaschphasen (im Bereich von 3 bis 60 Tagen) sind unter www. sportintegrity.ch/glukokortikoide publiziert und werden bei den relevanten Substanzen und Arzneimitteln in der Medikamentenabfrage Global DRO angezeigt.

\section{Handlungsanweisungen}

Werden die Auswaschphasen bei therapeutischer Anwendung vor einem Wettkampf eingehalten, so ist eine positive Dopingprobe im Wettkampf höchst unwahrscheinlich. Kann die Auswaschphase vor einem Wett-

Werden die Auswaschphasen eingehalten, ist eine positive Dopingprobe im Wettkampf höchst unwahrscheinlich.

kampf nicht eingehalten werden oder wird das Glukokortikoid im Wettkampf angewendet, sind die jeweiligen Handlungsanweisungen unter www.sportintegrity.ch/ glukokortikoide zu beachten.

In allen Situationen ist es für die Athletinnen und Athleten von grosser Wichtigkeit, dass Diagnosestellung und Therapiewahl durch die behandelnde Ärztin bzw. den behandelnden Arzt ausführlich begründet und dokumentiert werden.

Bildnachweis

Explorer Media Pty Ltd Sport The Library | Dreamstime.com

Literatur

1 Ventura R, Daley-Yates P, Mazzoni I, Collomp K, Saugy M, Buttgereit $\mathrm{F}$, et al. A novel approach to improve detection of glucocorticoid doping in sport with new guidance for physicians prescribing for athletes. Br J Sports Med. 2021 Apr 20. doi: 10.1136/ bjsports-2020-103512

2 www.wada-ama.org/sites/default/files/resources/files/2022list_ explanatory_note_final_en.pdf

\section{Das Wichtigste in Kürze}

- Die Stiftung Antidoping Schweiz heisst seit Anfang Jahr Swiss Sport Integrity. Sie übernimmt (neben den Anti-Doping-Aktivitäten) neu auch die Untersuchung von Meldungen zu Ethikverstössen.

- Dafür hat sie ein Meldeportal eingerichtet. Es steht allen Personen offen, die eine Meldung über mögliche Verstösse oder Missstände machen wollen.

- Die Welt-Anti-Doping-Agentur hat Anpassungen mit Auswirkungen auf den Dopingstatus von Arzneimitteln vorgenommen. Bei Glukokortikoiden sind neu jegliche Injektionen im Wettkampf verboten.

- Angaben zu den Auswaschphasen für die im Wettkampf verbotenen Glukokortikoide sind unter www.sportintegrity.ch/ glukokortikoide publiziert und werden in der Medikamentenabfrage Global DRO angezeigt. 